\title{
TOI-3362b: A Proto Hot Jupiter Undergoing High-eccentricity Tidal Migration
}

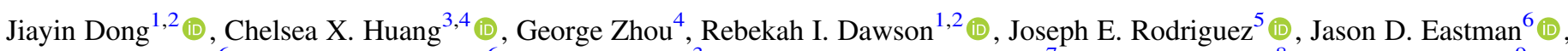

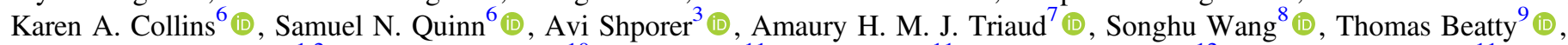
Jonathon M. Jackson ${ }^{1,2}$ (1), Kevin I. Collins ${ }^{10}{ }^{1}$, Lyu Abe $^{11}$, Olga Suarez $^{11}$ (i), Nicolas Crouzet ${ }^{12}$ (1), Djamel Mékarnia ${ }^{11}$ (i), Georgina Dransfield $^{7}$, Eric L. N. Jensen ${ }^{13}$ (1) , Chris Stockdale $^{14}(\mathbb{1})$, Khalid Barkaoui ${ }^{15,16}{ }^{(1)}$, Alexis Heitzmann ${ }^{4}(1)$,

Duncan J. Wright $^{4}\left(\mathbb{D}\right.$, Brett C. Addison ${ }^{4}$ (1), Robert A. Wittenmyer ${ }^{4}$ (1), Jack Okumura $^{4}$, Brendan P. Bowler ${ }^{17}$ (D),

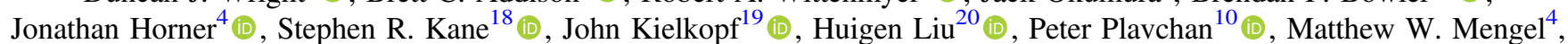

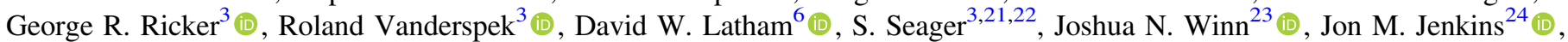
Jessie L. Christiansen ${ }^{25}$ (10), and Martin Paegert ${ }^{6}$ (1)

${ }^{1}$ Department of Astronomy \& Astrophysics, 525 Davey Laboratory, The Pennsylvania State University, University Park, PA, 16802, USA; jdong@psu.edu

${ }^{2}$ Center for Exoplanets and Habitable Worlds, 525 Davey Laboratory, The Pennsylvania State University, University Park, PA, 16802, USA

${ }^{3}$ Department of Physics and Kavli Institute for Astrophysics and Space Research, Massachusetts Institute of Technology, Cambridge, MA 02139, USA

${ }^{4}$ University of Southern Queensland, Centre for Astrophysics, West Street, Toowoomba, QLD 4350 Australia

${ }^{5}$ Department of Physics and Astronomy, Michigan State University, East Lansing, MI 48824, USA

${ }_{7}^{6}$ Center for Astrophysics | Harvard \& Smithsonian, 60 Garden Street, Cambridge, MA 02138, USA

${ }^{7}$ School of Physics \& Astronomy, University of Birmingham, Edgbaston, Birmingham B15 2TT, UK

${ }^{8}$ Department of Astronomy, Indiana University, Bloomington, IN 47405, USA

${ }^{9}$ Department of Astronomy and Steward Observatory, University of Arizona, Tucson, AZ 85721, USA

${ }^{10}$ George Mason University, 4400 University Drive MS 3F3, Fairfax, VA, 22030 USA

${ }^{11}$ Université Côte d'Azur, Observatoire de la Côte d'Azur, CNRS, Laboratoire Lagrange, Bd de l'Observatoire, CS 34229, F-06304 Nice cedex 4, France

${ }_{12}$ European Space Agency (ESA), European Space Research and Technology Centre (ESTEC), Keplerlaan 1, 2201 AZ Noordwijk, The Netherlands

${ }^{3}$ Department of Physics \& Astronomy, Swarthmore College, Swarthmore, PA 19081, USA

14 Hazelwood Observatory, Australia
19 C Allée du 6 Août, B-4000 Liége, Belgium

${ }^{16}$ Oukaimeden Observatory, High Energy Physics and Astrophysics Laboratory, Cadi Ayyad University, Marrakech, Morocco

${ }^{17}$ Department of Astronomy, The University of Texas at Austin, TX 78712, USA

${ }^{18}$ Department of Earth and Planetary Sciences, University of California, Riverside, CA 92521, USA

19 Department of Physics and Astronomy, University of Louisville, Louisville, KY 40292, USA

${ }^{20}$ School of Astronomy and Space Science, Key Laboratory of Modern Astronomy and Astrophysics in Ministry of Education, Nanjing University, Nanjing 210046 , Jiangsu, People's Republic of China

${ }^{21}$ Department of Earth, Atmospheric, and Planetary Sciences, Massachusetts Institute of Technology, Cambridge, MA 02139, USA

22 Department of Aeronautics and Astronautics, Massachusetts Institute of Technology, Cambridge, MA 02139, USA

${ }^{23}$ Department of Astrophysical Sciences, Princeton University, 4 Ivy Lane, Princeton, NJ 08544, USA

${ }^{24}$ NASA Ames Research Center, Moffett Field, CA 94035, USA

${ }^{25}$ Caltech/IPAC-NASA Exoplanet Science Institute, Pasadena, CA 91125, USA

Received 2021 July 5; revised 2021 September 8; accepted 2021 September 11; published 2021 October 7

\begin{abstract}
High-eccentricity tidal migration is a possible way for giant planets to be placed in short-period orbits. If this happens often, one would expect to catch proto hot Jupiters on highly elliptical orbits undergoing high-eccentricity tidal migration. As of yet, few such systems have been discovered. Here, we introduce TOI-3362b (TIC464300749b), an 18.1 day, $5 M_{\text {Jup }}$ planet orbiting a main-sequence F-type star that is likely undergoing higheccentricity tidal migration. The orbital eccentricity is $0.815_{-0.032}^{+0.023}$. With a semimajor axis of $0.153{ }_{-0.003}^{+0.002}$ au, the planet's orbit is expected to shrink to a final orbital radius of $0.051_{-0.006}^{+0.008}$ au after complete tidal circularization. Several mechanisms could explain the extreme value of the planet's eccentricity, such as planet-planet scattering and secular interactions. Such hypotheses can be tested with follow-up observations of the system, e.g., measuring the stellar obliquity and searching for companions in the system with precise, long-term radial-velocity observations. The variation in the planet's equilibrium temperature as it orbits the host star and the tidal heating at periapse make this planet an intriguing target for atmospheric modeling and observation. Because the planet's orbital period of 18.1 days is near the limit of TESS's period sensitivity, even a few such discoveries suggest that proto hot Jupiters may be quite common.
\end{abstract}

Unified Astronomy Thesaurus concepts: Exoplanet astronomy (486); Hot Jupiters (753); Transit photometry (1709); Radial velocity (1332); Exoplanet migration (2205)

\section{Introduction}

High-eccentricity tidal migration has long been proposed to explain the existence of close-in giant planets. In this scenario, a giant planet is formed several au away from its host star. Its orbital eccentricity is excited to a high value, perhaps via planet-planet scattering (e.g., Rasio \& Ford 1996; Chatterjee et al. 2008; Nagasawa et al. 2008) or secular interactions, such as von Zeipel-Lidov-Kozai oscillations (e.g., von Zeipel 1910; Kozai 1962; Lidov 1962; Wu \& Murray 2003; see Naoz 2016 for a review) and secular chaos (e.g., Wu \& Lithwick 2011). The orbit loses energy due to tidal dissipation as the planet approaches the star near periapse. Once the planet decouples from its perturber, the planet's orbital angular momentum is expected to be conserved (i.e., $L=M_{p} \sqrt{G M_{\star} a_{p}\left(1-e_{p}^{2}\right)}$ is 
constant). As a consequence, the initially cold planet migrates inward while its orbit circularizes, following the track $a_{p}\left(1-e_{p}^{2}\right)=$ constant $=a_{\text {final }}$. The final orbital radius of planet $a_{\text {final }}$ depends on the planet's initial semimajor axis and eccentricity. The tidal circularization timescale $\tau_{\mathrm{c}}$ has a strong dependence on $a_{\text {final }}$ (e.g., $\tau_{\mathrm{c}} \propto a_{\text {final }}^{8}$; Eggleton et al. 1998; Eggleton \& Kiseleva-Eggleton 2001; Hansen 2010) and therefore, only cold planets with sufficiently small $a_{\text {final }}$ could possibly circularize during the system's lifetime.

If close-in giant planets are products of high-eccentricity tidal migration, we would expect to catch at least some of them on the tidal circularization track (e.g., Socrates et al. 2012). For example, a planet tidally migrating to $a_{\text {final }}=0.05$ au will have an eccentricity of 0.86 when it is at $0.2 \mathrm{au}$. As of yet, few close-in giant planets have been observed with such extreme eccentricities and sufficiently small $a_{\text {final }}$ (i.e., $a_{\text {final }} \lesssim 0.05 \mathrm{au}$; Dawson et al. 2015). HD 80606b $\left(a_{p}=0.46 \mathrm{au}, e_{p}=0.93\right.$; Naef et al. 2001) might be the most impressive example of a proto hot Jupiter undergoing high-eccentricity tidal migration to be a hot Jupiter. The planet's distant stellar companion suggests stellar Kozai cycles as the possible mechanism to excite its extreme eccentricity (Wu \& Murray 2003). HAT-P-2b (HD 147506b; $a_{p}=0.068 \mathrm{au,}$ $e_{p}=0.52$; Bakos et al. 2007), which has a less extreme eccentricity, could be another example of a proto hot Jupiter undergoing tidal migration and circularization. Long-term radialvelocity observations of the system reveal the presence of a substellar companion (Lewis et al. 2013), also suggesting the stellar Kozai mechanism as the source of the eccentricity excitation. For planets with lower masses, Kepler-1656b $\left(a_{p}=\right.$ $0.20 \mathrm{au}, e_{p}=0.84, M_{p}=48 M_{\oplus}$; Brady et al. 2018) is an extraordinary example. The high physical density of the planet, along with the extreme eccentricity, might suggest planet-planet interactions such as collision and scattering in the planet's dynamical history.

In this work, we introduce TOI-3362b (TIC-464300749b, 2MASS J10235624-5650353), a transiting proto hot Jupiter that is also likely undergoing high-eccentricity tidal circularization. TOI-3362b was first identified by a systematic search for Warm Jupiters in the Southern Ecliptic Hemisphere in Year 1 of the TESS Full-Frame Images data (Dong et al. 2021). The unusually short duration of the transits compared to the expected duration for a circular orbit (e.g., Dawson \& Johnson 2012) pointed to its high eccentricity. Ground-based follow-up observations presented here validate the planet, break the degeneracy between the eccentricity and argument of periapse, and constrain the planet's mass.

In Section 2, we describe the TESS and ground-based photometric and spectroscopic follow-up observations of the target by ASTEP, LCOGT-SSO, CHIRON, and MinervaAustralis. In Section 3, we fit a model to determine the stellar and planetary parameters. In Section 4, we discuss the dynamical implications and propose further investigations of the system.

\section{Observations}

Here we describe the TESS photometry in Section 2.1, ground-based transit photometry by ASTEP and LCOGT-SSO (Brown et al. 2013) in Section 2.2, and ground-based spectroscopic observations by CHIRON and Minerva-Australis in Section 2.3.

\subsection{TESS Photometry}

TOI-3362 (TIC-464300749) was observed by TESS with a 30 minute cadence during Sectors 9 and 10 of its primary mission (2019 February 28 to 2019 April 22), and with a 10 minute cadence during Sectors 36 and 37 of its first extended mission (2021 March 07 to 2021 April 28). During a systematic search for warm, large planets using Quick-Look Pipeline lightcurves derived from TESS primary Full-Frame Images (FFIs; Huang et al. 2020a, 2020b; Dong et al. 2021), we detected a candidate transit signal at a period of 18.134 days, with signal-to-pink-noise (i.e., $1 / f$ frequency noise) ratio of 22.7. Three transits of TOI$3362 \mathrm{~b}$ were observed during the TESS primary mission. Preliminary analysis showed that the stellar density ratio $\rho_{\text {circ }} / \rho_{\star}$, where $\rho_{\text {circ }}$ is the inferred stellar density from the lightcurves assuming a circular orbit and $\rho_{\star}$ is from isochrone fitting, was about 18 (i.e., much larger than 1), and therefore the planetary candidate is highly likely to be on an eccentric orbit (Dong et al. 2014). Here we use early released TESS Image CAlibrator High Level Science Product (HLSP) FFIs (Fausnaugh et al. 2020) in the extended mission to derive the 10 minute cadence lightcurves. The method we employ is similar to that used to derive the standard QLP lightcurves. Three additional transits are observed. We show the raw and detrended lightcurves in Figure 1. The lightcurves are detrended using a Matern-3/2 Gaussian Process (GP) kernel (Foreman-Mackey et al. 2017; Foreman-Mackey 2018). There are no TESS spacecraft events impacting any of the transits. The second transit in the Year 3 data has a relatively high level of noise since it occurred at the beginning of a TESS orbit. We perform the light-curve fit with and without the second transit in Year 3 and find similar planetstar radius ratio posteriors. See Section 3.2 for more details.

\subsection{Ground-based Transit Photometry}

We used the TESS Transit Finder, which is a customized version of the Tapir software package (Jensen 2013), to schedule our transit observations. We observed three transits as part of the TESS Follow-up Observing Program. The detrended lightcurves from these observations can all be found on the ExoFOP-TESS website. ${ }^{26}$

The Antarctica Search for Transiting ExoPlanets (ASTEP) program observed two transits on the nights of UT 2020 August 10 and UT 2020 August 28 (Guillot et al. 2015; Mékarnia et al. 2016). The $0.4 \mathrm{~m}$ telescope is equipped with an FLI Proline science camera with a KAF-16801E, 40964096 front-illuminated $\mathrm{CCD}$ and is located on the east Antarctic plateau. The non-filtered red science channel is similar to the $R_{c}$ band in transmission. The first transit was observed in an 11".2 aperture including a neighboring star 3.3 magnitude fainter than TOI-3362. The second transit was observed in a 10 "' 1 aperture. The estimated transit depths from both transits are consistent with the TESS observations.

A full transit of TOI-3362b was observed in the Pan-STARSS $z_{s}$ and Bessell $B$ band on UT 2021 February 7 using a $1.0 \mathrm{~m}$ telescope at the Las Cumbres Observatory Global Telescope (LCOGT; Brown et al. 2013) Siding Spring Observatory (SSO) node in New South Wales, Australia. The LCOGT observations were calibrated with the standard BANZAI pipeline, and the lightcurves were extracted using AstroImage J (Collins et al. 2017). The observation used an uncontaminated 3." 5 aperture for

\footnotetext{
${ }^{26}$ https://exofop.ipac.caltech.edu/tess/
} 


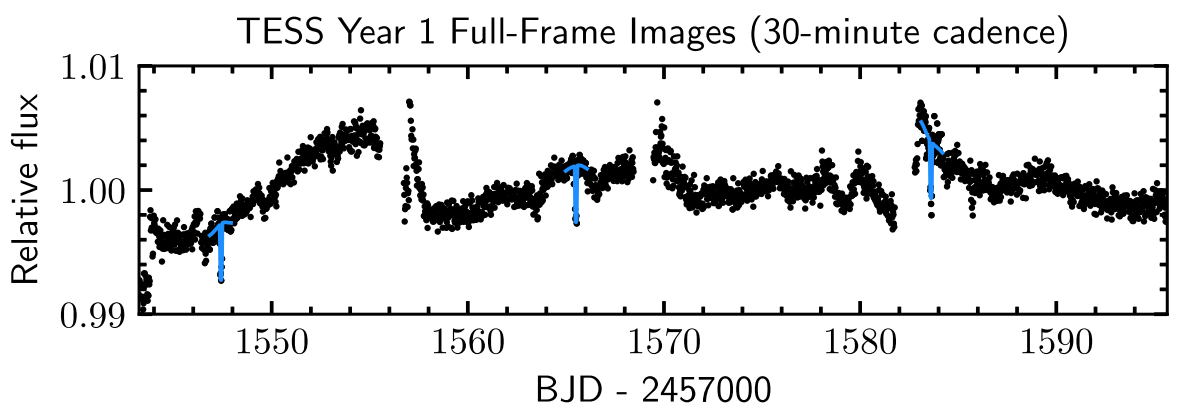

TESS Year 3 Full-Frame Images (10-minute cadence)

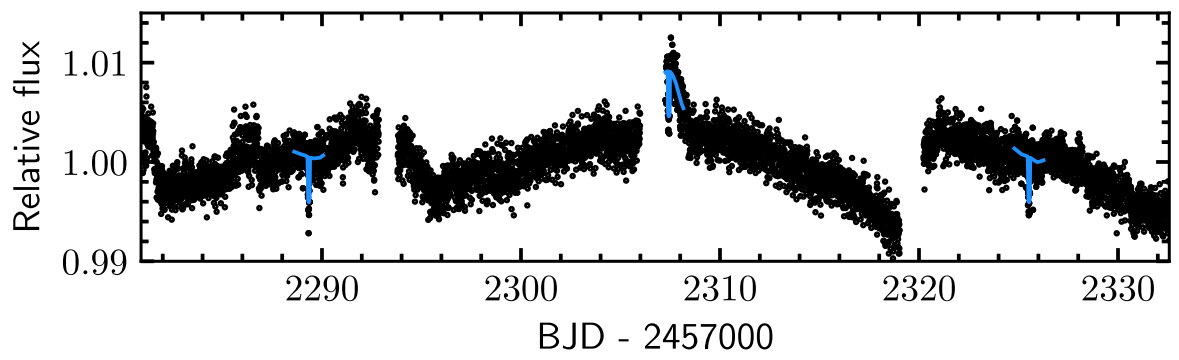

ASTEP Rc 2020.8.10

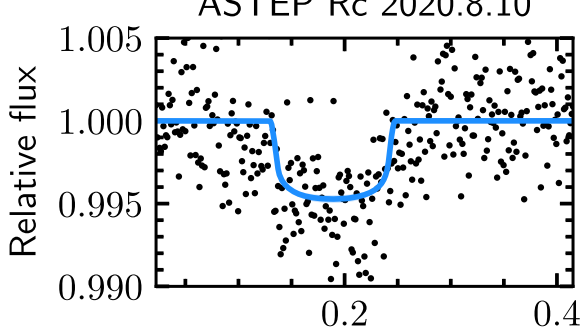

BJD - 2459072

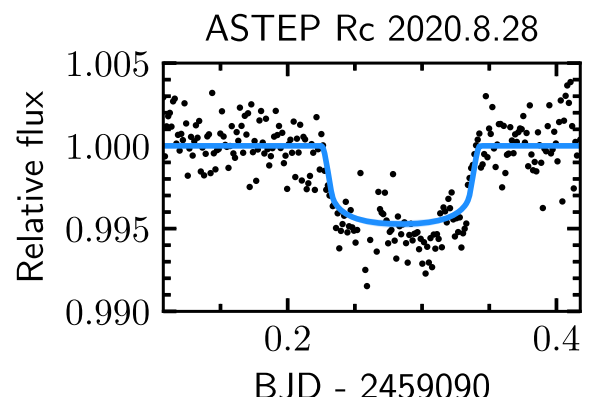

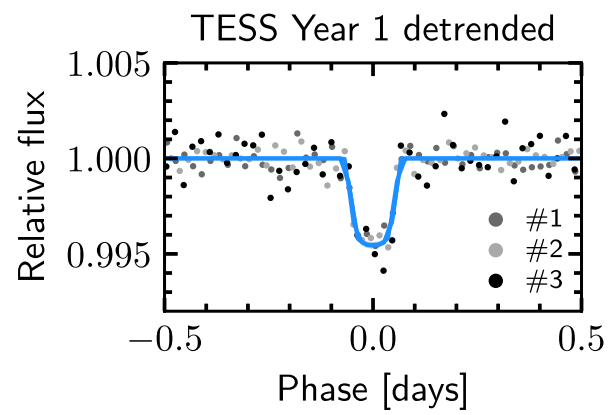

TESS Year 3 detrended

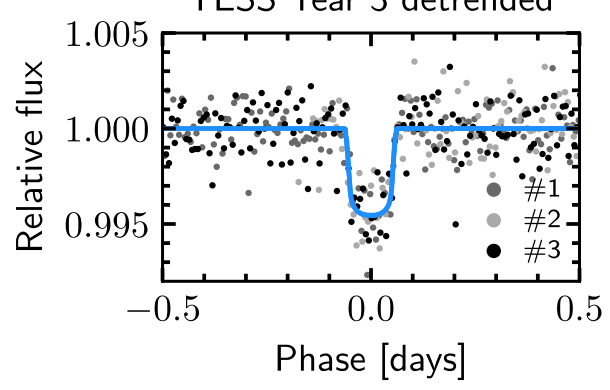

LCOGT-SSO B/zs 2021.2.7

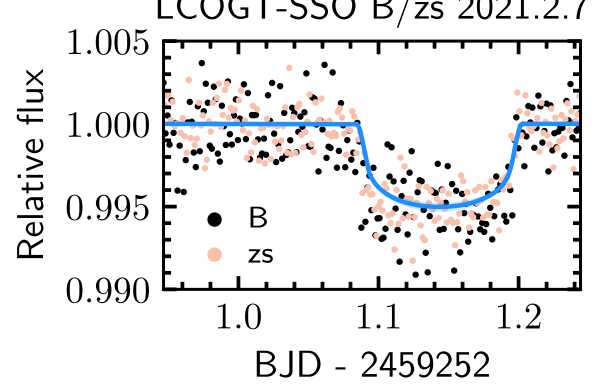

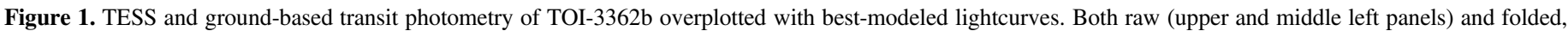

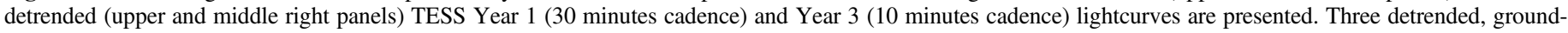

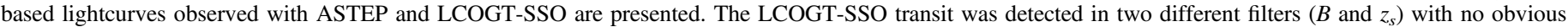
transit depth variation detected.

the $z_{s}$ and a 4 !" 67 aperture for the $B$ band and recovered the expected transit signal. The transit depths from the two bands show no strong chromaticity.

\subsection{Spectroscopic Observations}

Spectroscopic follow-up observations of TOI-3362 were conducted to measure the orbit and mass of the transiting planet and to refine the atmospheric parameters of the host star. We obtained 21 spectra using the CHIRON echelle spectrograph (Tokovinin et al. 2013) on the SMARTS $1.5 \mathrm{~m}$ telescope located at the Cerro Tololo Inter-American Observatory (CTIO), Chile, between UT 2021 April 15 and 2021 May 12. CHIRON is a high-resolution echelle spectrograph fed via an image slicer and a fiber bundle, with a resolving power of $R \approx 80,000$ over the wavelength range of $4100-8700 \AA$. The wavelength solution is provided via Th-Ar hollow-cathode lamp exposures that were taken after each science exposure. The spectra were extracted via the official CHIRON pipeline (Tokovinin et al. 2013). The radial velocities were derived as per the procedure described in Zhou et al. (2020). Briefly, we derived a line-broadening profile for each spectrum using a least-squares deconvolution (Donati et al. 1997) between the observed spectra and synthetic nonrotating spectral templates generated via the ATLAS9 stellar models (Castelli \& Hubrig 2004). The line-broadening profiles were fit via a convolution of the rotational, macroturbulent, and instrumental broadening kernels, yielding the radial-velocity shift and the rotational broadening velocity. To derive spectroscopic atmospheric parameters for TOI-3362, we match the CHIRON spectra against an observed spectral library that has been classified by the Spectral Classification Pipeline (Buchhave et al. 2012), following the procedure described in Zhou et al. (2020). We measure a spectroscopic effective temperature of $T_{\text {eff }}=6532_{-86}^{+88} \mathrm{~K}$, surface gravity $\log g=4.072_{-0.034}^{+0.032}$ dex, and bulk metallicity $[\mathrm{M} / \mathrm{H}]=0.017_{-0.049}^{+0.057} \mathrm{dex}$. The star has a stellar type of F5V.

We also obtained nine observations of TOI-3362 between 2021 May 16 and 2021 May 30 using the Minerva-Australis telescope array (Addison et al. 2019), located at Mt. Kent Observatory, Australia. Minerva-Australis is an array of four identical $0.7 \mathrm{~m}$ telescopes linked via fiber feeds to a single KiwiSpec echelle spectrograph at a spectral resolving power of $R \approx 80,000$ over the wavelength region of $5000-6300 \AA$. The array is wholly dedicated to radial-velocity follow-up of TESS planet candidates (e.g., Nielsen et al. 2019; Brahm et al. 2020; Addison et al. 2021). Simultaneous wavelength calibration is provided via two calibration fibers illuminated by a quartz lamp through an iodine cell. The spectra were extracted for each telescope individually and the radial velocities were extracted via the same techniques as 
those described above for the CHIRON observations. We note, though, that due to throughput issues, only velocities from Telescope 1 of the MINERVA-Australis array were adopted for this analysis; the remaining telescopes yielded velocity uncertainties too large to contribute meaningfully to the orbit detection.

\section{Results}

Here we describe models to infer the stellar and planetary parameters. In Section 3.1, we present the stellar isochrone and spectral energy distribution (SED) fitting results. In Section 3.2, we show the transit-only model and report the transit-timing variation analysis and planet's eccentricity inferred purely from lightcurves. In Section 3.4, we describe the transit and radialvelocity joint model and present the planetary parameters.

\subsection{Stellar Properties}

We used the exoplanet fitting suite EXOFASTV2 (Eastman et al. 2013, 2019) to perform a fit of the SED for TOI-3362. We combined the available Gaia $G, B_{P}$, and $R_{P}$, (Gaia Collaboration et al. 2018), 2MASS $J_{2 M}, H_{2 M}$, and $K_{S, 2 M}$ (Cutri et al. 2003), and WISE W1 and W2 (Zacharias et al. 2017) photometry with Gaussian priors on the metallicity $(0.002 \pm 0.070), T_{\text {eff }}(6513 \pm$ $100 \mathrm{~K}$ ) from spectroscopy (see Section 2.3), and the Gaia DR2 parallax $(2.7265 \pm 0.03952 \mathrm{mas}$, corrected for the $-30 \mu \mathrm{as}$ offset reported by Lindegren et al. 2018). We also placed an upper limit on the line-of-sight extinction of 4.1844 mag from Schlegel et al. (1998) and Schlafly \& Finkbeiner (2011). Within the fit, the MESA Isochrones and Stellar Tracks (MIST) stellar evolution models (Choi et al. 2016; Dotter 2016) are used to provide better estimates for the host-star parameters. We also put a lower bound on the age of the host star of $100 \mathrm{Myr}$ since we do not see any signs of youth in the photometry or spectroscopy (e.g., from the stellar rotation period and lithium abundance). TIC-464300749 has a mass of $1.445_{-0.073}^{+0.069} M_{\odot}$, a radius of $1.830_{-0.053}^{+0.055} R_{\odot}$, and an age of $2.14_{-0.52}^{+0.66}$ Gyr. See Table 1 for a complete list of the results from this analysis.

We also calculated the stellar density and radius from isochrone fitting with the Dartmouth (Dotter et al. 2008) stellar evolution models. The approach-as described by Dawson et al. (2015) - fits the stellar effective temperature, metallicity, Gaia DR2 parallax, and Gaia apparent $g$ magnitude (Gaia Collaboration et al. 2016, 2018), where the stellar temperature, metallicity, and uncertainties are inferred from the CHIRON spectra. We applied the systematic correction to Gaia parallaxes from Stassun \& Torres (2018). The values derived from the Dartmouth model are consistent with the MIST model and have similar uncertainties.

\subsection{Transit-only Model}

The TESS and ground-based transit photometry have been described in Sections 2.1 and 2.2 and are shown in Figure 1. We only model TESS lightcurves around each transit to roughly six times the transit duration to reduce computational expense. We use a quadratic limb-darkening transit model (Mandel \& Agol 2002; Kipping 2013) plus a Matern-3/2 GP kernel with a white-noise term to model the lightcurves (Foreman-Mackey et al. 2017; Foreman-Mackey 2018). We use informative limb-darkening coefficients from Claret (2017) for transits in different filters (TESS, $R_{c}, B, z_{s}$ ). TESS Year 1 observations, Year 3 observations, and each of the three ground-based observations have their own GP kernels to account for differences in variability captured by different wavelengths, instruments, and cadences. The stellar density $\rho_{\text {circ }}$ is modeled assuming the planet has a circular orbit. We later compare the marginalized $\rho_{\text {circ }}$ to $\rho_{\star}$, the stellar density derived from the isochrone fitting, to constrain the planet's eccentricity. To characterize the transit-timing variation signal of the planet, we model the mid-transit times $T_{1 . . N}$ individually. All free parameters in the transit-only model are

$$
\begin{aligned}
& \left\{\rho_{\text {circ }}, b, r_{p} / r_{\star}, T_{1 . . N},\right. \\
& \quad u_{0 . .1, \mathrm{TESS}}, u_{0 . .1, R c}, u_{0 . .1, B}, u_{0 . .1, z_{\mathrm{s}}}, \\
& s_{\mathrm{TESS}, \mathrm{Y} 1}, \rho_{\mathrm{TESS}, \mathrm{Y} 1}, \sigma_{\mathrm{TESS}, \mathrm{Y} 1} \\
& s_{\mathrm{TESS}, \mathrm{Y} 3}, \rho_{\mathrm{TESS}, \mathrm{Y} 3}, \sigma_{\mathrm{TESS}, \mathrm{Y} 3} \\
& s_{\mathrm{ASTEP}, 1}, \rho_{\mathrm{ASTEP}, 1}, \sigma_{\mathrm{ASTEP}, 1} \\
& s_{\mathrm{ASTEP}, 2}, \rho_{\mathrm{ASTEP}, 2}, \sigma_{\mathrm{ASTEP}, 2} \\
& \left.s_{\mathrm{LCOGT}}, \rho_{\mathrm{LCOGT}}, \sigma_{\mathrm{LCOGT}}\right\}
\end{aligned}
$$

where $\rho_{\text {circ }}$ is the stellar density assuming a circular orbit, $b$ is the impact parameter, $r_{p} / r_{\star}$ is the planet-star radius ratio, $u_{0 . .1}$ are the quadratic limb-darkening coefficients, and $s$ is the photometric white noise. The Matern-3/2 GP kernel follows $\mathcal{K}(\tau)=\sigma^{2}(1+\sqrt{3} \tau / \rho) \exp (-\sqrt{3} \tau / \rho)$, where $\sigma$ represents the amplitude of variability and $\rho$ represents the timescale. The priors used in this model are the same as the ones listed in Table 2 of Dong et al. (2021).

We use the exoplanet package (Foreman-Mackey et al. 2021) for the light-curve fits. Four chains are sampled using the Markov Chain Monte Carlo (MCMC) technique with gradientbased proposals (Hoffman \& Gelman 2011; Neal 2012; Betancourt 2017). Each chain contains 50,000 tuning steps and 3000 sampling steps with a target accept rate of 0.95 . We assess MCMC convergence using the summary statistics, e.g., Gelman-Rubin diagnostic ( $\hat{\mathcal{R}}<1.1$ for convergence), trace plots, and corner plots (Foreman-Mackey 2016) of the marginal joint distributions. The $\hat{\mathcal{R}}$ values for our model parameters are all less than 1.001 by the end of the sampling. All four chains are combined to get the posteriors.

We use the medians and $1 \sigma$ uncertainties of the mid-transit time posteriors to perform a least-square fit to a linear line. The orbital period and the conjunction time of the transiting planet are inferred. The $O-C$ residuals, which are calculated by subtracting linear ephemerides from observed mid-transit times, present no significant transit-timing variations. We can rule out any level of transit-timing variability greater than $\sim 5$ minutes.

From the stellar density posteriors $\rho_{\text {circ }}$ and $\rho_{\star}$, we infer the eccentricity $e$ and argument of periapse $\omega$ of the planet, shown as the black histograms and contours in Figure 2. The stellar densities suggest the planet is on a highly elliptical orbit.

\subsection{Transit-only Model with EXOFASTv2}

For a consistency check, we also modeled the TESS lightcurve and ground-based transits, along with the stellar model described in Section 3.1 and including planetary eccentricity directly, using EXOFASTV2 (Eastman et al. 2019).

We used keplerspline from Vanderburg \& Johnson (2014) to account for the long-term variability of the TESS lightcurves and averaged 10 model data points over the 30 minute Sectors 9 and 10 exposures, and 4 model data points over the 10 minute Sectors 36 and 37 exposures. We also fit a dilution term to the TESS lightcurves to account for poor 
Table 1

Median Values and 68\% Confidence Intervals for the Stellar and Planetary Parameters of TOI-3362b

\begin{tabular}{|c|c|c|c|c|}
\hline Parameter & Units & Values & & \\
\hline \multicolumn{5}{|l|}{ Stellar Parameters ${ }^{\mathrm{a}}$} \\
\hline$M_{*}$ & $\operatorname{Mass}\left(M_{\odot}\right)$ & $1.445_{-0.073}^{+0.069}$ & & \\
\hline$R_{*}$ & Radius $\left(R_{\odot}\right)$ & $1.830_{-0.053}^{+0.055}$ & & \\
\hline$L_{*}$ & Luminosity $\left(L_{\odot}\right)$ & $5.48_{-0.32}^{+0.37}$ & & \\
\hline$\rho_{*}$ & Density (cgs) & $0.331_{-0.032}^{+0.035}$ & & \\
\hline $\log g$ & Surface gravity (cgs) & $4.072_{-0.034}^{+0.032}$ & & \\
\hline$T_{\text {eff }}$ & Effective temperature $(\mathrm{K})$ & $6532_{-86}^{+88}$ & & \\
\hline$[\mathrm{M} / \mathrm{H}]$ & Bulk metallicity (dex) & $0.017_{-0.049}^{+0.057}$ & & \\
\hline$v \sin I_{\star}$ & Rotational line broadening $\left(\mathrm{km} \mathrm{s}^{-1}\right)$ & $20.0 \pm 4.1$ & & \\
\hline Age & Age $(\mathrm{Gyr})$ & $2.14_{-0.52}^{+0.66}$ & & \\
\hline EEP & Equal evolutionary phase $^{\mathrm{b}}$ & $369_{-19}^{+22}$ & & \\
\hline$A_{V}$ & $V$-band extinction (mag) & $0.098_{-0.056}^{+0.062}$ & & \\
\hline$\sigma_{\mathrm{SED}}$ & SED photometry error scaling & $0.50_{-0.11}^{+0.18}$ & & \\
\hline$\varpi$ & Parallax (mas) & $2.724_{-0.039}^{+0.040}$ & & \\
\hline$G$ & Gaia $G$ magnitude & $10.705 \pm 0.020$ & & \\
\hline$B_{\mathrm{P}}$ & Gaia $B_{\mathrm{P}}$ magnitude & $10.948 \pm 0.020$ & & \\
\hline$R_{\mathrm{P}}$ & Gaia $R_{\mathrm{P}}$ magnitude & $10.335 \pm 0.020$ & & \\
\hline$J$ & 2MASS $J$ magnitude & $9.944 \pm 0.024$ & & \\
\hline$H$ & 2MASS $H$ magnitude & $9.719 \pm 0.022$ & & \\
\hline$K_{\mathrm{S}}$ & 2MASS $K_{\mathrm{S}}$ magnitude & $9.693 \pm 0.023$ & & \\
\hline WISE1 & WISE1 magnitude & $9.651 \pm 0.030$ & & \\
\hline WISE2 & WISE2 magnitude & $9.678 \pm 0.030$ & & \\
\hline WISE3 & WISE3 magnitude & $9.643 \pm 0.063$ & & \\
\hline WISE4 & WISE4 magnitude & $9.427 \pm 0.516$ & & \\
\hline \multicolumn{5}{|c|}{ Planetary Parameters (joint model) } \\
\hline$P$ & Period (days) & $18.09547_{-0.00003}^{+0.00003}$ & & \\
\hline$T_{C}$ & Mid-transit time (BJD-2,457,000) & $1529.325_{-0.001}^{+0.001}$ & & \\
\hline$b$ & Transit impact parameter & $0.270_{-0.184}^{+0.207}$ & & \\
\hline$\delta$ & Transit depth & $0.0041_{-0.0002}^{+0.0002}$ & & \\
\hline$M_{P}$ & Mass $\left(M_{\mathrm{Jup}}\right)$ & $5.029_{-0.646}^{+0.668}$ & & \\
\hline$R_{P}$ & Radius $\left(R_{\mathrm{Jup}}\right)$ & $1.142_{-0.041}^{+0.043}$ & & \\
\hline$a$ & Semimajor axis (au) & $0.153_{-0.003}^{+0.002}$ & & \\
\hline$i$ & Inclination $\left(^{\circ}\right)$ & $89.140_{-0.668}^{+0.584}$ & & \\
\hline$e$ & Eccentricity & $0.815_{-0.032}^{+0.023}$ & & \\
\hline$\omega$ & Argument of periapse $\left(^{\circ}\right)$ & $50.873_{-9.165}^{+11.085}$ & & \\
\hline \multicolumn{2}{|l|}{ Instrumental Parameters } & $u_{0}$ & $u_{1}$ & \\
\hline$u_{\text {TESS }}$ & Limb-darkening coefficients & $0.18_{-0.09}^{+0.09}$ & $0.30_{-0.10}^{+0.10}$ & \\
\hline$u_{\mathrm{Rc}}$ & Limb-darkening coefficients & $0.23_{-0.10}^{+0.10}$ & $0.39_{-0.09}^{+0.10}$ & \\
\hline$u_{B}$ & Limb-darkening coefficients & $0.46_{-0.10}^{+0.10}$ & $0.30_{-0.10}^{+0.10}$ & \\
\hline \multirow[t]{2}{*}{$u_{z \mathrm{~s}}$} & Limb-darkening coefficients & $0.13_{-0.10}^{+0.10}$ & $0.38_{-0.10}^{+0.10}$ & \\
\hline & & $s(\mathrm{ppt})$ & $\rho$ (days) & $\sigma(\mathrm{ppt})$ \\
\hline TESS, Y1 & Gaussian Process parameters & $0.71_{-0.04}^{+0.04}$ & $3.11_{-1.18}^{+2.32}$ & $3.83_{-1.16}^{+2.27}$ \\
\hline TESS, Y3 & Gaussian Process parameters & $1.25_{-0.04}^{+0.04}$ & $2.28_{-0.71}^{+1.25}$ & $4.19_{-1.13}^{+2.18}$ \\
\hline ASTEP, 1 & Gaussian Process parameters & $5.49_{-0.01}^{+0.02}$ & $4.58_{-4.45}^{+181.50}$ & $0.59_{-0.46}^{+1.23}$ \\
\hline ASTEP, 2 & Gaussian Process parameters & $5.49_{-0.01}^{+0.03}$ & $22.80_{-21.57}^{+281.40}$ & $2.29_{-1.15}^{+4.47}$ \\
\hline \multirow[t]{2}{*}{ LCOGT } & Gaussian Process parameters & $5.50_{-0.02}^{+0.03}$ & $2.55_{-2.53}^{+155.36}$ & $0.18_{-0.13}^{+0.56}$ \\
\hline & & CHIRON & Minerva & \\
\hline$\gamma_{\text {rel }}$ & Relative RV offset $\left(\mathrm{m} \mathrm{s}^{-1}\right)$ & $6450_{-37}^{+36}$ & $7617_{-62}^{+60}$ & \\
\hline$\sigma_{J}$ & $\mathrm{RV}$ jitter $\left(\mathrm{m} \mathrm{s}^{-1}\right)$ & $3_{-3}^{+31}$ & $5_{-5}^{+50}$ & \\
\hline$k_{\mathrm{bkg}}$ & Radial velocity baseline $\left(\mathrm{m} \mathrm{s}^{-1}\right.$ day $\left.^{-1}\right)$ & $0_{-3}^{+4}$ & $-1_{-7}^{+7}$ & \\
\hline
\end{tabular}

Notes.

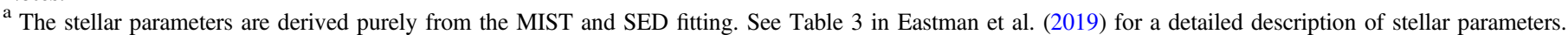

${ }^{\mathrm{b}}$ Corresponds to static points in a star's evolutionary history; see Section 2 in Dotter (2016). 


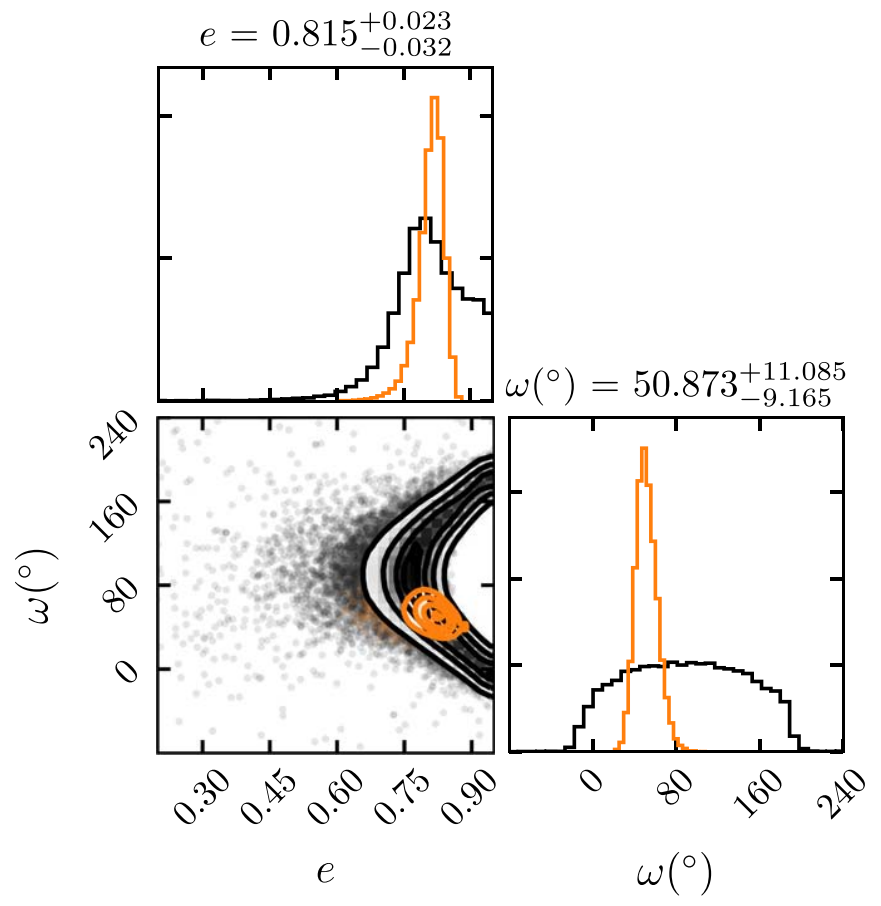

Figure 2. Joint posterior distributions of the eccentricity and argument of periapse for TOI-3362b. Values inferred purely from the "photoeccentric" effect are colored in black and from the transit $+\mathrm{RV}$ joint fit in orange. The contours are $0.5,1,1.5$, and 2 sigma levels. Radial-velocity measurements confirm the extreme eccentricity of TOI- $3362 \mathrm{~b}$ and provide much tighter constraints.

background-subtraction (Burt et al. 2020). In practice, fitting the dilution term means the transit depth is defined by the ground-based lightcurves.

The stellar model therefore constrains the stellar density, and the transit duration then constrains the eccentricity.

Most of our values were consistent with the results shown in Section 3.2. In particular, our eccentricity was within $0.05 \sigma$. The worst agreement was in our fractional depth which we measured as $R_{P} / R_{*}=0.0685_{-0.0010}^{+0.0012}-2.3 \sigma$ discrepant, likely because of the different detrending methods.

\subsection{Joint Transit and Radial-velocity Modeling}

Here we combine the photometry with ground-based spectroscopic observations, as described in Section 2.3, to infer the planet's mass and put a tighter constraint on the eccentricity. Since the planet shows no evidence of transit-timing variations, we directly model its orbital period and conjunction time. The priors of these values follow normal distributions with medians of 18.1 days and 2,458,529.3 BJD, respectively, and uncertainties of 0.1 days.

The joint model has the parameters mentioned in the transitonly model but also includes planet mass $M_{p}$, orbital eccentricity $e$, and argument of periapse $\omega$. Only the stellar density was modeled in the transit-only analysis. To jointly fit the radial-velocity data, we will also need the stellar mass. Picking two parameters out of $\rho_{\star}, M_{\star}$, and $R_{\star}$ will be sufficient. We choose $M_{\star}$ and $R_{\star}$ for the joint model since the pair shows little covariance in the stellar-fit posteriors discussed in Section 3.1. The stellar-fit posteriors are used as the priors for $M_{\star}$ and $R_{\star}$ in the joint model. To account for the instrumentation offsets and systematics, both CHIRON and MinervaAustralis are given an additive offset parameter and a lognormal radial-velocity jitter term. We also include a linear function of time for both instruments as a baseline model for stellar activity.

We use the exoplanet package for the joint fit, following the same sampling and post-analysis procedures as described in Section 3.2. No significant background trends are found in CHIRON and Minerva-Australis observations. A summary of the posteriors can be found in Table 1. The best-modeled lightcurves and radial-velocity orbit can be found in Figures 1 and 3 , respectively.

\section{Discussion and Future Work}

The inferred semimajor axis and eccentricity of TOI-3362b are consistent with a proto hot Jupiter that is undergoing higheccentricity tidal migration and will have an orbital radius of $0.051_{-0.006}^{+0.008}$ au (i.e., an orbital period of $3.526_{-0.584}^{+0.823}$ days) after complete tidal circularization (see Section 3.2 of Dawson \& Johnson 2018 for a detailed discussion). The timescale for the circularization can be estimated using the equilibrium tide model (Hut 1981), although the estimation has significant uncertainty due to the poorly understood tidal dissipation parameter $Q^{\prime}{ }_{p}$ and how it depends on the planet's physical and orbital properties. Neglecting stellar tidal dissipation and assuming that the planet rotates synchronously with its orbit, we adopt Equation (3) in Adams \& Laughlin (2006) and find a characteristic circularization timescale of $2.7 \mathrm{Gyr}$ for TOI-3362b, using $Q^{\prime}{ }_{p}=10^{6}$. We note the estimated timescale could differ by orders of magnitude for different $Q^{\prime}{ }_{p}$ assumptions. Moreover, the $Q^{\prime}{ }_{p}$ is not a constant during planetary evolution, but has a strong dependence on the planetary radius and semimajor axis. If we instead assume a constant viscous dissipation rate $\bar{\sigma}_{p}$ (Eggleton et al. 1998; Eggleton \& Kiseleva-Eggleton 2001; Hansen 2010) and adopt Equations (2), (3), and (6) in Hansen (2010), we find the circularization timescale to be $4 \mathrm{Gyr}$, using $\bar{\sigma}_{p}=5 \times 10^{-6}$. Again, different assumptions for $\bar{\sigma}_{p}$ could lead to a very different estimation of the circularization timescale. TOI-3362 has an estimated age of $2.14_{-0.52}^{+0.66} \mathrm{Gyr}$, and is expected to leave the main sequence (MS) and begin its post-MS radial expansion in $\sim 0.5 \mathrm{Gyr}$ according to the MIST model. Comparing the host-star's lifetime to the planet's circularization timescale, it is very plausible that TOI- $3362 \mathrm{~b}$ could not be fully circularized before its host star evolves, even if it acquired its eccentricity early on. If the planet's orbit has not yet been fully circularized, the increasing stellar radiation could inflate the planetary radius and speed up the orbit circularization. After the orbital circularization, the tide raised on the star could introduce stellar dissipation and the orbital decay of the newly formed hot Jupiter. Post-MS evolution of the host star (e.g., stellar radial expansion and mass loss) could introduce orbital instability for TOI-3362b, especially if the system has planetary or stellar companions (e.g., Veras et al. 2013, 2017; Stephan et al. 2018), or lead to engulfment by the host star (e.g., Metzger et al. 2012; Stephan et al. 2020).

In a broad context, in Figure 4, we present the eccentricity versus semimajor axis for all confirmed close-in planets with wellconstrained eccentricities (i.e., mass $<13 M_{\text {Jup }}$, period $<365$ days) taken from the NASA Exoplanet Archive (DOI 10.26133/NEA12) as of 2021 July 26. The gray region indicates the parameter space in which planets from a large initial semimajor axis could have undergone high-eccentricity tidal migration following the constant angular momentum tracks (i.e., $a_{\text {final }}=a\left(1-e^{2}\right)=$ constant). The upper boundary of the gray region is the limit for avoiding tidal disruption earlier on the migration track. When the initial orbit at the beginning of tidal circularization is highly elliptical, the initial 


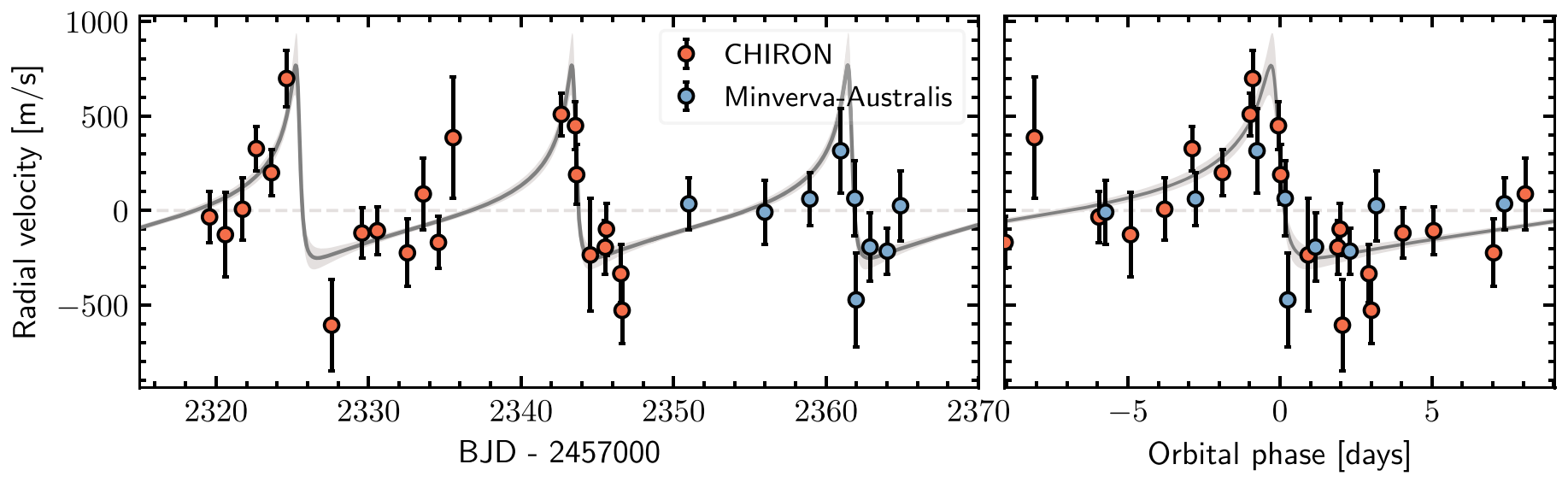

Figure 3. Radial-velocity observations of TOI-3362 by CHIRON colored in orange and Minerva-Australis colored in blue. The instrumental offsets and linear background trends have been abstracted from CHIRON and Minerva-Australis data. The phased radial velocity is plotted in the right panel, where the planet transits at $t=0$. The median and $1 \sigma$ uncertainty of the planetary radial-velocity signal are shown in gray.

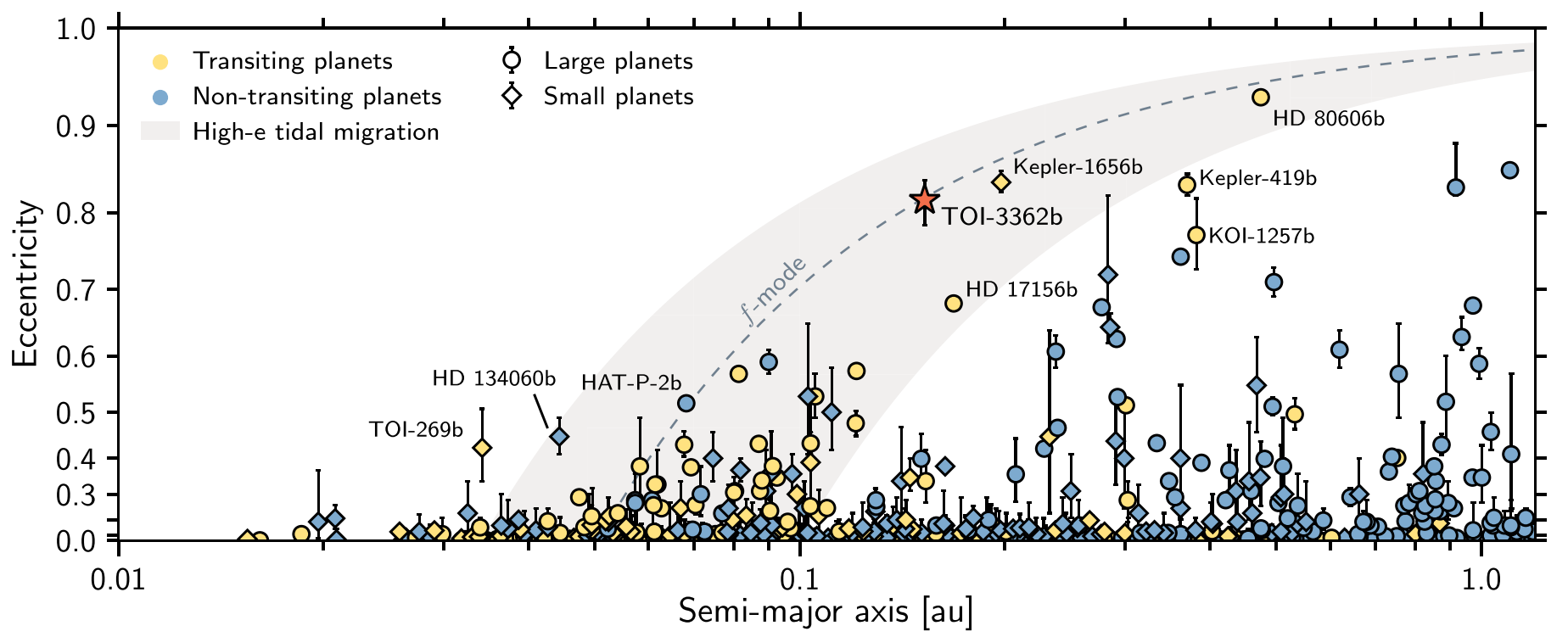

Figure 4. Eccentricity versus semimajor axis for all confirmed planets less than 13 Jupiter-mass with an orbital period less than 365 days. Data are extracted from the NASA Exoplanet Archive (DOI 10.26133/NEA12) as of 2021 July 26. The $y$-axis is scaled to $e^{2}$ to emphasize non-circular planets. The $x$-axis is in log scale. The gray region with a final semimajor axis range of $0.034-0.1$ au is set by the Roche limit and the tidal circularization timescale, respectively. Planets above the dashed gray line could have their $f$-mode tidal dissipation excited to speed up the migration. Transiting planets are labeled in yellow and non-transiting planets are labeled in blue. Large planets $\left(R_{p}>6 R_{\oplus}\right.$ and/or $\left.M_{p}>100 M_{\oplus}\right)$ are labeled as circles and small planets $\left(R_{p}<6 R_{\oplus}\right.$ and /or $\left.M_{p}<100 M_{\oplus}\right)$ are labeled as diamonds. To help with the interpretation, we only plot planets with well-constrained eccentricities, which are planets with eccentricity uncertainties less than $50 \%$ of the measured eccentricities or uncertainties less than 0.2 for those on nearly circular orbit $(e<0.2)$.

periapse $\approx \frac{1}{2} a_{\text {final }}$ because tracks of constant angular momentum are defined by $a_{\text {final }}=a\left(1-e^{2}\right)$ and $a\left(1-e^{2}\right) \approx 2 a(1-e)$ for $e \rightarrow 1$. Therefore, only planets with $a_{\text {final }}=2 f_{p} R_{p}\left(M_{\star} / M_{p}\right)^{1 / 3}$ or greater, where $f_{p}=2.7$ (Guillochon et al. 2011), could have avoided tidal disruption during periapse passages. For illustrative purposes, we assume a $1 M_{\text {Jup }}, 1.3 R_{\text {Jup }}$ planet orbiting a solar-mass star and derive $a_{\text {final }}=a\left(1-e^{2}\right)=0.034$ au, set as the upper boundary of the gray region. The lower boundary of the gray region is set by the tidal circularization timescale. Planets below the region are unlikely to get tidally circularized within their systems' lifetimes. The critical limit for $a_{\text {final }}$ depends on the tidal dissipation efficiency, which is not well determined. For illustrative purposes, we adopt the value $a_{\text {final }}=a\left(1-e^{2}\right)=0.1$ au, set as the lower boundary of the gray region. For planets that are formed at large semimajor axes and have been undergoing high-eccentricity tidal migration, we would expect to observe them only in the gray region if they are decoupled from their perturber. We also show the constant angular momentum track for planets with periapses small enough that they could excite planetary $f$-mode dissipation (Wu 2018; Vick et al. 2019). Above the dashed line, the planet's periapse is close enough to the host star to excite $f$-mode oscillation which leads to rapid orbital decay from a "cold" Jupiter to a "warm" Jupiter, so we expect an absence of highly elliptical Jupiters (i.e., $e>0.9$ ) above that line. We adopt the criterion in $\mathrm{Wu}$ (2018), where $a_{\text {final }}=2 f_{p} R_{p}\left(M_{\star} / M_{p}\right)^{1 / 3}$ and $f_{p} \approx 4$, and derive $a_{\text {final }}=a\left(1-e^{2}\right)=0.05$ au for a $1 M_{\text {Jup }}, 1.3 R_{\text {Jup }}$ planet orbiting a solar-mass star.

TOI-3362b stands out because of its extreme eccentricity. Although many giant planets in the semimajor axis range of 0.1-1 au have non-circular orbits, only a few have eccentricities high enough to be consistent with the high-eccentricity tidal migration origin. TOI-3362b joins HD 80606b (Naef et al.2001; 
Wu \& Murray 2003) as an example of proto hot Jupiters with an extreme eccentricity, suggesting high-eccentricity tidal migration as one possible origin of hot Jupiters. In Figure 4, we label a few additional interesting systems. The $y$-axis in Figure 4 is scaled to $e^{2}$ to emphasize planets with non-circular orbits. Beside HD 80606b, HAT-P-2b, and Kepler-1656b which have been discussed in Section 1, HD 17156b (Fischer et al. 2007) could undergo tidal migration if its tidal dissipation is efficient enough. Planets below the gray region (e.g., Kepler-419b, Dawson et al. 2014; Jackson et al. 2019; KOI-1257b, Santerne et al. 2014) could periodically reach the migration track if they are coupled to external companions that cyclically excite their eccentricities to higher values.

In Figure 4, we also observe that nearly all the planets to the left of the dashed gray line have been fully circularized, which may indicate that tidal circularization happens very quickly at $a_{\text {final }}<0.05$ au. Two exceptions are TOI-269b (Cointepas et al. 2021) and HD 134060b (Udry et al. 2019), which are both small planets and could have much longer tidal evolution timescales compared to giant planets. For giant planets, the $f$ mode dissipation could aid the migration during the early stage, e.g., from 1-0.2 au with an eccentricity of 0.98-0.9. Although the $f$-mode line separates the population, the $f$-mode dissipation is likely not responsible for the lack of planets with $0.2<e<0.9$ since it only works efficiently when the planet has a very large eccentricity.

A group of hot Jupiters on moderately elliptical orbits (i.e., $0.2<e<0.6)$ are found in the gray region with $a_{\text {final }}$ of $0.05-0.1 \mathrm{au}$, but not many on extremely elliptical orbits. Many of these moderately elliptical planets were discovered with ground-based transit surveys that are not sensitive to longer orbital periods, which might explain why we do not see these moderately eccentric Jupiters' highly eccentric counterparts earlier on the tidal migration track. However, in the Kepler sample-which includes longer orbital periods-Dawson et al. (2015) found a paucity of super-eccentric proto hot Jupiters inconsistent with the prediction of the high-eccentricity tidal migration origin of close-in giant planets (Socrates et al. 2012), assuming a similar share of the Kepler hot Jupiter population has moderately elliptical orbits as the hot Jupiters found in ground-based surveys. Recent work suggests that for planets undergoing the von Zeipel-Lidov-Kozai cycles, their extreme eccentricity spike is very short (e.g., Naoz et al. 2011; Teyssandier et al. 2013) and their transition timescale from cold Jupiters to hot Jupiters is short compared to the timescale the planets spend as cold Jupiters (Petrovich 2015a; Anderson \& Lai 2017), making migrating planets hard to detect. A similar short transition timescale is also found in mechanisms such as secular chaos (e.g., Teyssandier et al. 2019) and planet-planet scattering (e.g., Chatterjee et al. 2008; Nagasawa et al. 2008). However, none of these studies could explain the lack of supereccentric planets $(e>0.6)$ relative to moderately eccentric planets $(0.2<e<0.6$; Dawson et al. 2015). One proposed explanation is that planets undergoing tidal migration primarily orbit more metal-rich (i.e., greater than or equal to solar metallicity) stars (Dawson \& Murray-Clay 2013), so the Kepler sample lacks both moderately eccentric hot Jupiters and supereccentric proto hot Jupiters due to the Kepler host stars' overall lower metallicity compared to ground-based surveys (Dawson \& Murray-Clay 2013; Guo et al. 2017). TOI-3362 has a metallicity of $[\mathrm{M} / \mathrm{H}]=0.017_{-0.049}^{+0.057} \mathrm{dex}$, consistent with the picture of planets undergoing tidal migration, primarily those orbiting metal-rich stars. We have not computed a formal prediction for the number of super-eccentric Jupiters in the TESS sample, but would have not expected many due to the shorter orbital periods likely to be detected by the TESS mission given the observing strategy. The discovery of TOI$3362 \mathrm{~b}$ right at the upper limit of TESS planets' most common orbital periods suggests that proto hot Jupiters may not be as rare among bright, nearby stars as they are in the Kepler sample. A final possibility is that hot Jupiters may begin as warm Jupiters instead of cold Jupiters, following in situ formation or disk migration.

Several dynamical processes could explain the extreme eccentricity of TOI-3362b. Generally for Jupiters on the higheccentricity tidal migration track, the possible dynamical processes include planet-planet scattering (e.g., Rasio \& Ford 1996; Chatterjee et al. 2008; Nagasawa et al. 2008), stellar/planetary Kozai cycles with tidal friction (e.g., Wu \& Murray 2003; Naoz et al. 2012; Petrovich 2015b) and secular chaos (e.g., Wu \& Lithwick 2011). The stellar metallicity trend (Dawson \& Murray-Clay 2013) supports mechanisms involving planet-planet interactions (e.g., planet-planet scattering, secular chaos, and planetary Kozai). The short observing baseline and limited radial-velocity precision hinder the detection of radial-velocity accelerations in the TOI-3362 system. From the Gaia EDR3 astrometry (Lindegren et al. 2021), the astrometric noise excess significance is 6.38 , which indicates moderate astrometric excess noise. However, the renormalized unit weight error (RUWE) value of TOI-3362 is 0.929 , which indicates the excess noise is unlikely to be from orbital motion. The low RUWE value and the fact that Gaia did not report any additional source within $5^{\prime \prime}$ of TOI-3362 can be used to place some limited constraint on the mass of bound companions within 1.5-6.5 au (Penoyre et al. 2020) as well as beyond $\sim 30$ au (Rizzuto et al. 2018; Ziegler et al. 2018). Future Gaia astromeric limits with longer baselines will help to improve such a constraint. Long-term, precise radial-velocity observations will be key to searching for companions in the TOI-3362 system. High-resolution speckle imaging will be helpful to identify or rule out nearby binaries. Excitation of the mutual inclination might also happen during dynamical interactions. The planet is at wide enough separation that it is presumably not realigned by stellar tides. A RossiterMcLaughlin measurement would, therefore, help to reveal the dynamical history of the planet.

The extreme eccentricity of TOI-3362b makes it an exciting target for atmospheric observations. The incident flux received by TOI-3362b at periapse is $\sim 80$ times higher than the flux it receives at apoapse. The equilibrium temperature at periapse is $\sim 2500 \mathrm{~K}$, comparable to classic super Hot Jupiters such as WASP-121 (Delrez et al. 2016; Evans et al. 2017). At apoapse, the equilibrium temperature is $\sim 800 \mathrm{~K}$. As the planet cools down, its atmosphere will cross the equal abundance boundaries of $\mathrm{CO} / \mathrm{CH}_{4}$ and $\mathrm{N}_{2} / \mathrm{NH}_{3}$ (Fortney et al. 2020). Significant condensation of clouds is also expected due to the dramatic temperature change (Wakeford et al. 2017). TOI-3362b provides an extreme case for studies of dynamic exoplanet atmosphere chemistry. The extreme time varying irradiation would also result in strong super-rotating jets in the atmosphere of TOI-3362b. When observed in relatively long wavelengths near the periapse of the orbit, we expect significant flux change (i.e., Laughlin et al. 2009; Mayorga et al. 2021) and a "ringing" effect due to the planet's rotation (Kataria et al. 2013). These flux variations can be 
easily measured by the MIRI instrument on the James Webb Space Telescope and lead to the direct detection of the rotation period of the planet. TOI-3362b may also experience significant tidal heating due to its high eccentricity. Adopting the tidal heating model in Leconte et al. (2010), we estimate the tidal energy dissipation rate is $10^{28} \mathrm{erg} \mathrm{s}^{-1}$, which is $10 \%$ of the orbitaveraged irradiation energy the planet receives from the star. Future atmosphere observations exploring the pressure-temperature profile of the planet may reveal the impact of such tidal heating.

We thank the referee for a helpful report and in particular for comments that improved the discussion of the work. We thank Alex Venner for a helpful discussion on the interpretation of the Gaia astrometry.

This research made use of exoplanet (Foreman-Mackey et al. 2019, 2021) and its dependencies (Astropy Collaboration et al. 2013, 2018; Kipping 2013; Salvatier et al. 2016; Theano Development Team 2016; Foreman-Mackey et al. 2017, 2019; Foreman-Mackey 2018; Luger et al. 2019; Agol et al. 2020). Computations for this research were performed on the Pennsylvania State University's Institute for CyberScience Advanced CyberInfrastructure (ICS-ACI). This content is solely the responsibility of the authors and does not necessarily represent the views of the Institute for CyberScience. The Center for Exoplanets and Habitable Worlds is supported by the Pennsylvania State University and the Eberly College of Science.

This work makes use of observations from the LCOGT network. Part of the LCOGT telescope time was granted by NOIRLab through the Mid-Scale Innovations Program (MSIP). MSIP is funded by NSF.

This research has used data from the CTIO/SMARTS $1.5 \mathrm{~m}$ telescope, which is operated as part of the SMARTS Consortium by RECONS (www.recons.org) members Todd Henry, Hodari James, Wei-Chun Jao, and Leonardo Paredes. At the telescope, observations were carried out by Roberto Aviles and Rodrigo Hinojosa.

Data presented herein were obtained with the MINERVAAustralis facility at the Mt. Kent Observatory from telescope time allocated through the NN-EXPLORE program. NNEXPLORE is a scientific partnership of the National Aeronautics and Space Administration and the National Science Foundation.

The CTIO/SMARTS $1.5 \mathrm{~m}$ and MINERVA-Australis telescope time were granted by the NOIRLab program 2021A0147 (PI: J. Dong).

This work has made use of data from the European Space Agency (ESA) mission Gaia (https://www.cosmos.esa.int/ gaia), processed by the Gaia Data Processing and Analysis Consortium (DPAC, https://www.cosmos.esa.int/web/gaia/ dpac/consortium). Funding for the DPAC has been provided by national institutions, in particular the institutions participating in the Gaia Multilateral Agreement.

We acknowledge the use of TESS High-Level Science Products (HLSP) produced by the Quick-Look Pipeline (QLP) at the TESS Science Office at MIT, which are publicly available from the Mikulski Archive for Space Telescopes (MAST). Funding for the TESS mission is provided by NASA's Science Mission directorate.
This paper includes data collected by the TESS mission, which are publicly available from the Mikulski Archive for Space Telescopes (MAST). Resources supporting this work were provided by the NASA High-End Computing (HEC) Program through the NASA Advanced Supercomputing (NAS) Division at Ames Research Center for the production of the SPOC data products.

This research has made use of the NASA Exoplanet Archive, which is operated by the California Institute of Technology, under contract with the National Aeronautics and Space Administration under the Exoplanet Exploration Program.

This research has made use of the Exoplanet Follow-up Observation Program website, which is operated by the California Institute of Technology, under contract with the National Aeronautics and Space Administration under the Exoplanet Exploration Program.

This research received funding from the European Research Council (ERC) under the European Union's Horizon 2020 research and innovation program (grant agreement $n^{\circ} 803193$ / BEBOP), and from the Science and Technology Facilities Council (STFC; grant $\mathrm{n}^{\circ}$ ST/S00193X/1).

This work makes use of observations from the ASTEP telescope. ASTEP benefited from the support of the French and Italian polar agencies IPEV and PNRA in the framework of the Concordia station program and from Idex UCAJEDI (ANR-15IDEX-01).

MINERVA-Australis is supported by Australian Research Council LIEF grant LE160100001, Discovery grant DP180100972, Mount Cuba Astronomical Foundation, and institutional partners University of Southern Queensland, UNSW Sydney, MIT, Nanjing University, George Mason University, University of Louisville, University of California Riverside, University of Florida, and The University of Texas at Austin. We respectfully acknowledge the traditional custodians of all lands throughout Australia, and recognise their continued cultural and spiritual connection to the land, waterways, cosmos, and community. We pay our deepest respects to all Elders, ancestors and descendants of the Giabal, Jarowair, and Kambuwal nations, upon whose lands the MinervaAustralis facility at Mt Kent is situated.

We gratefully acknowledge support by NASA XRP 80NSSC18K0355 and NASA TESS GO 80NSSC18K1695.

Facility: TESS, Gaia, LCOGT, ASTEP, CTIO: $1.5 \mathrm{~m}$ CHIRON, Minerva-Australis, Exoplanet Archive.

Software: Arviz (Kumar et al. 2019), AstroImageJ (Collins et al. 2017), astropy (Astropy Collaboration et al. 2013, 2018), celerite (Foreman-Mackey et al. 2017; Foreman-Mackey 2018), exoplanet (Foreman-Mackey et al. 2021), Jupyter (Kluyver et al. 2016), Matplotlib (Hunter 2007; Droettboom et al. 2016), NumPy (van der Walt et al. 2011; Harris et al. 2020), pandas (McKinney 2010), PyMC3 (Salvatier et al. 2016), Scipy (Virtanen et al. 2020), starry (Luger et al. 2019), TAPIR (Jensen 2013), Theano (Theano Development Team 2016), EXOFASTv2 (Eastman et al. 2013, 2019).

\section{Appendix \\ Radial Velocities for TOI-3362}

In Table 2, we present radial-velocity data obtained from the CHIRON and Minerva-Australis spectrographs. 
Table 2

Radial Velocities for TOI-3362

\begin{tabular}{lccc}
\hline \hline BJD & RV $\left(\mathrm{m} \mathrm{s}^{-1}\right)$ & $\sigma_{\mathrm{RV}}\left(\mathrm{m} \mathrm{s}^{-1}\right)$ & Instrument \\
\hline $2,459,319.576$ & 6423.3 & 135.8 & CHIRON \\
$2,459,320.613$ & 6329.0 & 224.7 & CHIRON \\
$2,459,321.737$ & 6463.0 & 165.2 & CHIRON \\
$2,459,322.642$ & 6783.3 & 118.6 & CHIRON \\
$2,459,323.634$ & 6655.6 & 123.0 & CHIRON \\
$2,459,324.630$ & 7153.7 & 148.8 & CHIRON \\
$2,459,327.580$ & 5846.3 & 242.0 & CHIRON \\
$2,459,329.568$ & 6333.2 & 132.9 & CHIRON \\
$2,459,330.579$ & 6344.9 & 127.8 & CHIRON \\
$2,459,332.550$ & 6226.9 & 179.4 & CHIRON \\
$2,459,333.592$ & 6538.0 & 190.0 & CHIRON \\
$2,459,334.584$ & 6281.0 & 137.0 & CHIRON \\
$2,459,335.551$ & 6835.0 & 321.0 & CHIRON \\
$2,459,342.644$ & 6955.0 & 114.0 & CHIRON \\
$2,459,343.571$ & 6894.5 & 127.8 & CHIRON \\
$2,459,343.648$ & 6635.2 & 158.0 & CHIRON \\
$2,459,344.548$ & 6209.6 & 299.3 & CHIRON \\
$2,459,345.525$ & 6249.9 & 143.0 & CHIRON \\
$2,459,345.601$ & 6345.5 & 137.8 & CHIRON \\
$2,459,346.545$ & 6109.4 & 152.6 & CHIRON \\
$2,459,346.628$ & 5915.4 & 176.0 & CHIRON \\
\hline $2,459,351.004$ & 7667.0 & 137.0 & Minerva-Australis \\
$2,459,355.986$ & 7613.0 & 170.0 & Minerva-Australis \\
$2,459,358.939$ & 7677.0 & 141.0 & Minerva-Australis \\
$2,459,360.963$ & 7928.0 & 223.0 & Minerva-Australis \\
$2,459,361.889$ & 7674.0 & 199.0 & Minerva-Australis \\
$2,459,361.974$ & 7137.0 & 250.0 & Minerva-Australis \\
$2,459,362.885$ & 7415.0 & 183.0 & Minerva-Australis \\
$2,459,364.020$ & 7391.0 & 123.0 & Minerva-Australis \\
$2,459,364.887$ & 7630.0 & 186.0 & Minerva-Australis \\
\hline & & &
\end{tabular}

\section{ORCID iDs}

Jiayin Dong (1) https://orcid.org/0000-0002-3610-6953 Chelsea X. Huang (i) https://orcid.org/0000-0003-0918-7484

Rebekah I. Dawson (1) https://orcid.org/0000-0001-9677-1296 Joseph E. Rodriguez (1) https://orcid.org/0000-00018812-0565

Jason D. Eastman (1) https://orcid.org/0000-0003-3773-5142 Karen A. Collins (i) https://orcid.org/0000-0001-6588-9574 Samuel N. Quinn (1) https://orcid.org/0000-0002-8964-8377 Avi Shporer (1) https://orcid.org/0000-0002-1836-3120 Amaury H. M. J. Triaud (1) https://orcid.org/0000-00025510-8751

Songhu Wang (1) https://orcid.org/0000-0002-7846-6981 Thomas Beatty (i) https://orcid.org/0000-0002-9539-4203 Jonathon M. Jackson (i) https://orcid.org/0000-00020323-4828

Kevin I. Collins (iD https://orcid.org/0000-0003-2781-3207 Olga Suarez (1) https://orcid.org/0000-0002-3503-3617 Nicolas Crouzet (1) https://orcid.org/0000-0001-7866-8738 Djamel Mékarnia (1) https://orcid.org/0000-0001-5000-7292 Eric L. N. Jensen (i) https://orcid.org/0000-0002-4625-7333 Chris Stockdale (10) https://orcid.org/0000-0003-2163-1437 Khalid Barkaoui (1) https://orcid.org/0000-0003-1464-9276 Alexis Heitzmann (i) https://orcid.org/0000-0002-8091-7526 Duncan J. Wright (1) https://orcid.org/0000-0001-7294-5386 Brett C. Addison (1) https://orcid.org/0000-0003-3216-0626 Robert A. Wittenmyer (1) https://orcid.org/0000-00019957-9304
Brendan P. Bowler (10 https://orcid.org/0000-0003-2649-2288 Jonathan Horner (1) https://orcid.org/0000-0002-1160-7970 Stephen R. Kane (1) https://orcid.org/0000-0002-7084-0529 John Kielkopf (1) https://orcid.org/0000-0003-0497-2651 Huigen Liu (1) https://orcid.org/0000-0001-5162-1753 Peter Plavchan (1) https://orcid.org/0000-0002-8864-1667 George R. Ricker 니 https://orcid.org/0000-0003-2058-6662 Roland Vanderspek 난 https://orcid.org/0000-0001-6763-6562 David W. Latham (즌 https://orcid.org/0000-0001-9911-7388 Joshua N. Winn (1) https://orcid.org/0000-0002-4265-047X Jon M. Jenkins (1) https://orcid.org/0000-0002-4715-9460 Jessie L. Christiansen (1) https://orcid.org/0000-00028035-4778

Martin Paegert (1) https://orcid.org/0000-0001-8120-7457

\section{References}

Adams, F. C., \& Laughlin, G. 2006, ApJ, 649, 1004

Addison, B., Wright, D. J., Wittenmyer, R. A., et al. 2019, PASP, 131, 115003 Addison, B. C., Wright, D. J., Nicholson, B. A., et al. 2021, MNRAS, 502, 3704

Agol, E., Luger, R., \& Foreman-Mackey, D. 2020, AJ, 159, 123

Anderson, K. R., \& Lai, D. 2017, MNRAS, 472, 3692

Astropy Collaboration, Price-Whelan, A. M., Sipőcz, B. M., et al. 2018, AJ, 156,123

Astropy Collaboration, Robitaille, T. P., Tollerud, E. J., et al. 2013, A\&A, 558, A33

Bakos, G. Á., Kovács, G., Torres, G., et al. 2007, ApJ, 670, 826

Betancourt, M. 2017, arXiv:1701.02434

Brady, M. T., Petigura, E. A., Knutson, H. A., et al. 2018, AJ, 156, 147

Brahm, R., Nielsen, L. D., Wittenmyer, R. A., et al. 2020, AJ, 160, 235

Brown, T. M., Baliber, N., Bianco, F. B., et al. 2013, PASP, 125, 1031

Buchhave, L. A., Latham, D. W., Johansen, A., et al. 2012, Natur, 486, 375

Burt, J. A., Nielsen, L. D., Quinn, S. N., et al. 2020, AJ, 160, 153

Castelli, F., \& Hubrig, S. 2004, A\&A, 425, 263

Chatterjee, S., Ford, E. B., Matsumura, S., \& Rasio, F. A. 2008, ApJ, 686, 580 Choi, J., Dotter, A., Conroy, C., et al. 2016, ApJ, 823, 102

Claret, A. 2017, A\&A, 600, A30

Cointepas, M., Almenara, J. M., Bonfils, X., et al. 2021, A\&A, 650, A145

Collins, K. A., Kielkopf, J. F., Stassun, K. G., \& Hessman, F. V. 2017, AJ, 153,77

Cutri, R. M., Skrutskie, M. F., van Dyk, S., et al. 2003, yCat, 2246, 0

Dawson, R. I., \& Johnson, J. A. 2012, ApJ, 756, 122

Dawson, R. I., \& Johnson, J. A. 2018, ARA\&A, 56, 175

Dawson, R. I., Johnson, J. A., Fabrycky, D. C., et al. 2014, ApJ, 791, 89

Dawson, R. I., \& Murray-Clay, R. A. 2013, ApJL, 767, L24

Dawson, R. I., Murray-Clay, R. A., \& Johnson, J. A. 2015, ApJ, 798, 66

Delrez, L., Santerne, A., Almenara, J. M., et al. 2016, MNRAS, 458, 4025

Donati, J. F., Semel, M., Carter, B. D., Rees, D. E., \& Collier Cameron, A. 1997, MNRAS, 291, 658

Dong, J., Huang, C. X., Dawson, R. I., et al. 2021, ApJS, 255, 6

Dong, S., Katz, B., \& Socrates, A. 2014, ApJL, 781, L5

Dotter, A. 2016, ApJS, 222, 8

Dotter, A., Chaboyer, B., Jevremović, D., et al. 2008, ApJS, 178, 89

Droettboom, M., Hunter, J., Caswell, T. A., et al. 2016, Matplotlib: Matplotlib V1.5.1, v1.5.1, Zenodo doi: $10.5281 /$ zenodo.44579

Eastman, J., Gaudi, B. S., \& Agol, E. 2013, PASP, 125, 83

Eastman, J. D., Rodriguez, J. E., Agol, E., et al. 2019, arXiv:1907.09480

Eggleton, P. P., Kiseleva, L. G., \& Hut, P. 1998, ApJ, 499, 853

Eggleton, P. P., \& Kiseleva-Eggleton, L. 2001, ApJ, 562, 1012

Evans, T. M., Sing, D. K., Kataria, T., et al. 2017, Natur, 548, 58

Fausnaugh, M. M., Burke, C. J., Ricker, G. R., \& Vanderspek, R. 2020, RNAAS, 4, 251

Fischer, D. A., Vogt, S. S., Marcy, G. W., et al. 2007, ApJ, 669, 1336

Foreman-Mackey, D. 2016, JOSS, 1, 24

Foreman-Mackey, D. 2018, RNAAS, 2, 31

Foreman-Mackey, D., Agol, E., Ambikasaran, S., \& Angus, R. 2017, AJ, 154,220

Foreman-Mackey, D., Czekala, I., Luger, R., et al. 2019, dfm/exoplanet: exoplanet v0.2.1, Zenodo, doi:10.5281/zenodo.3462740

Foreman-Mackey, D., Luger, R., Agol, E., et al. 2021, JOSS, 6, 3285

Fortney, J. J., Visscher, C., Marley, M. S., et al. 2020, AJ, 160, 288

Gaia Collaboration, Brown, A. G. A., Vallenari, A., et al. 2018a, A\&A, 616, A1 
Gaia Collaboration, Prusti, T., de Bruijne, J. H. J., et al. 2016, A\&A, 595, A1

Guillochon, J., Ramirez-Ruiz, E., \& Lin, D. 2011, ApJ, 732, 74

Guillot, T., Abe, L., Agabi, A., et al. 2015, AN, 336, 638

Guo, X., Johnson, J. A., Mann, A. W., et al. 2017, ApJ, 838, 25

Hansen, B. M. S. 2010, ApJ, 723, 285

Harris, C. R., Jarrod Millman, K., van der Walt, S. J., et al. 2020, arXiv:2006. 10256

Hoffman, M. D., \& Gelman, A. 2011, arXiv:1111.4246

Huang, C. X., Vanderburg, A., Pál, A., et al. 2020a, RNAAS, 4, 204

Huang, C. X., Vanderburg, A., Pál, A., et al. 2020b, RNAAS, 4, 206

Hunter, J. D. 2007, CSE, 9, 90

Hut, P. 1981, A\&A, 99, 126

Jackson, J. M., Dawson, R. I., \& Zalesky, J. 2019, AJ, 157, 166

Jensen, E. 2013, Tapir: A web interface for transit/eclipse observability, Astrophysics Source Code Library, ascl:1306.007

Kataria, T., Showman, A. P., Lewis, N. K., et al. 2013, ApJ, 767, 76

Kipping, D. M. 2013, MNRAS, 435, 2152

Kluyver, T., Ragan-Kelley, B., Pérez, F., et al. 2016, in Positioning and Power in Academic Publishing: Players, Agents and Agendas, ed. F. Loizides \& B. Scmidt (Amsterdam: IOS Press), 87

Kozai, Y. 1962, AJ, 67, 591

Kumar, R., Carroll, C., Hartikainen, A., \& Martin, O. 2019, JOSS, 4, 1143 Laughlin, G., Deming, D., Langton, J., et al. 2009, Natur, 457, 562

Leconte, J., Chabrier, G., Baraffe, I., \& Levrard, B. 2010, A\&A, 516, A64

Lewis, N. K., Knutson, H. A., Showman, A. P., et al. 2013, ApJ, 766, 95

Lidov, M. L. 1962, P\&SS, 9, 719

Lindegren, L., Hernández, J., Bombrun, A., et al. 2018, A\&A, 616, A2

Lindegren, L., Klioner, S. A., Hernández, J., et al. 2021, A\&A, 649, A2

Luger, R., Agol, E., Foreman-Mackey, D., et al. 2019, AJ, 157, 64

Mandel, K., \& Agol, E. 2002, ApJL, 580, L171

Mayorga, L. C., Robinson, T. D., Marley, M. S., May, E. M., \& Stevenson, K. B. 2021, arXiv:2105.08009

McKinney, W. 2010, in Proc. of the 9th Python in Science Conf., ed. S. van der Walt \& J. Millman (Austin, TX: SciPy), 56

Mékarnia, D., Guillot, T., Rivet, J. P., et al. 2016, MNRAS, 463, 45

Metzger, B. D., Giannios, D., \& Spiegel, D. S. 2012, MNRAS, 425, 2778

Naef, D., Latham, D. W., Mayor, M., et al. 2001, A\&A, 375, L27

Nagasawa, M., Ida, S., \& Bessho, T. 2008, ApJ, 678, 498

Naoz, S. 2016, ARA\&A, 54, 441
Naoz, S., Farr, W. M., Lithwick, Y., Rasio, F. A., \& Teyssandier, J. 2011, Natur, 473, 187

Naoz, S., Farr, W. M., \& Rasio, F. A. 2012, ApJL, 754, L36

Neal, R. M. 2012, arXiv:1206.1901

Nielsen, L. D., Bouchy, F., Turner, O., et al. 2019, A\&A, 623, A100

Penoyre, Z., Belokurov, V., Wyn Evans, N., Everall, A., \& Koposov, S. E. 2020, MNRAS, 495, 321

Petrovich, C. 2015a, ApJ, 805, 75

Petrovich, C. 2015b, ApJ, 799, 27

Rasio, F. A., \& Ford, E. B. 1996, Sci, 274, 954

Rizzuto, A. C., Vanderburg, A., Mann, A. W., et al. 2018, AJ, 156, 195

Salvatier, J., Wiecki, T. V., \& Fonnesbeck, C. 2016, PeerJ Computer Science, 2, e55

Santerne, A., Hébrard, G., Deleuil, M., et al. 2014, A\&A, 571, A37

Schlafly, E. F., \& Finkbeiner, D. P. 2011, ApJ, 737, 103

Schlegel, D. J., Finkbeiner, D. P., \& Davis, M. 1998, ApJ, 500, 525

Socrates, A., Katz, B., Dong, S., \& Tremaine, S. 2012, ApJ, 750, 106

Stassun, K. G., \& Torres, G. 2018, ApJ, 862, 61

Stephan, A. P., Naoz, S., \& Gaudi, B. S. 2018, AJ, 156, 128

Stephan, A. P., Naoz, S., Gaudi, B. S., \& Salas, J. M. 2020, ApJ, 889, 45

Teyssandier, J., Lai, D., \& Vick, M. 2019, MNRAS, 486, 2265

Teyssandier, J., Naoz, S., Lizarraga, I., \& Rasio, F. A. 2013, ApJ, 779, 166

Theano Development Team 2016, arXiv:1605.02688

Tokovinin, A., Fischer, D. A., Bonati, M., et al. 2013, PASP, 125, 1336

Udry, S., Dumusque, X., Lovis, C., et al. 2019, A\&A, 622, A37

van der Walt, S., Colbert, S. C., \& Varoquaux, G. 2011, CSE, 13, 22

Vanderburg, A., \& Johnson, J. A. 2014, PASP, 126, 948

Veras, D., Georgakarakos, N., Dobbs-Dixon, I., \& Gänsicke, B. T. 2017, MNRAS, 465, 2053

Veras, D., Mustill, A. J., Bonsor, A., \& Wyatt, M. C. 2013, MNRAS, 431, 1686 Vick, M., Lai, D., \& Anderson, K. R. 2019, MNRAS, 484, 5645

Virtanen, P., Gommers, R., Oliphant, T. E., et al. 2020, NatMe, 17, 261

von Zeipel, H. 1910, AN, 183, 345

Wakeford, H. R., Visscher, C., Lewis, N. K., et al. 2017, MNRAS, 464, 4247

Wu, Y. 2018, AJ, 155, 118

Wu, Y., \& Lithwick, Y. 2011, ApJ, 735, 109

Wu, Y., \& Murray, N. 2003, ApJ, 589, 605

Zacharias, N., Finch, C., \& Frouard, J. 2017, yCat, 1340

Zhou, G., Winn, J. N., Newton, E. R., et al. 2020, ApJL, 892, L21

Ziegler, C., Law, N. M., Baranec, C., et al. 2018, AJ, 156, 259 